$\frac{12 / 27-9508010}{3-70}$

UCRL-ID-117982

\title{
BEGET: The B-Factory Event Generator Version 21
}

\section{Douglas M. Wright}

August 1994

This is an informal report intended primarily for internal or limited external distribution. The opinions and conclusions stated are those of the author and may or may not be those of the Laboratory.

Work performed under the auspices of the U.S. Department of Energy by the Lawrence Livermore National Laboratory under Contract W-7405-Eng-48.

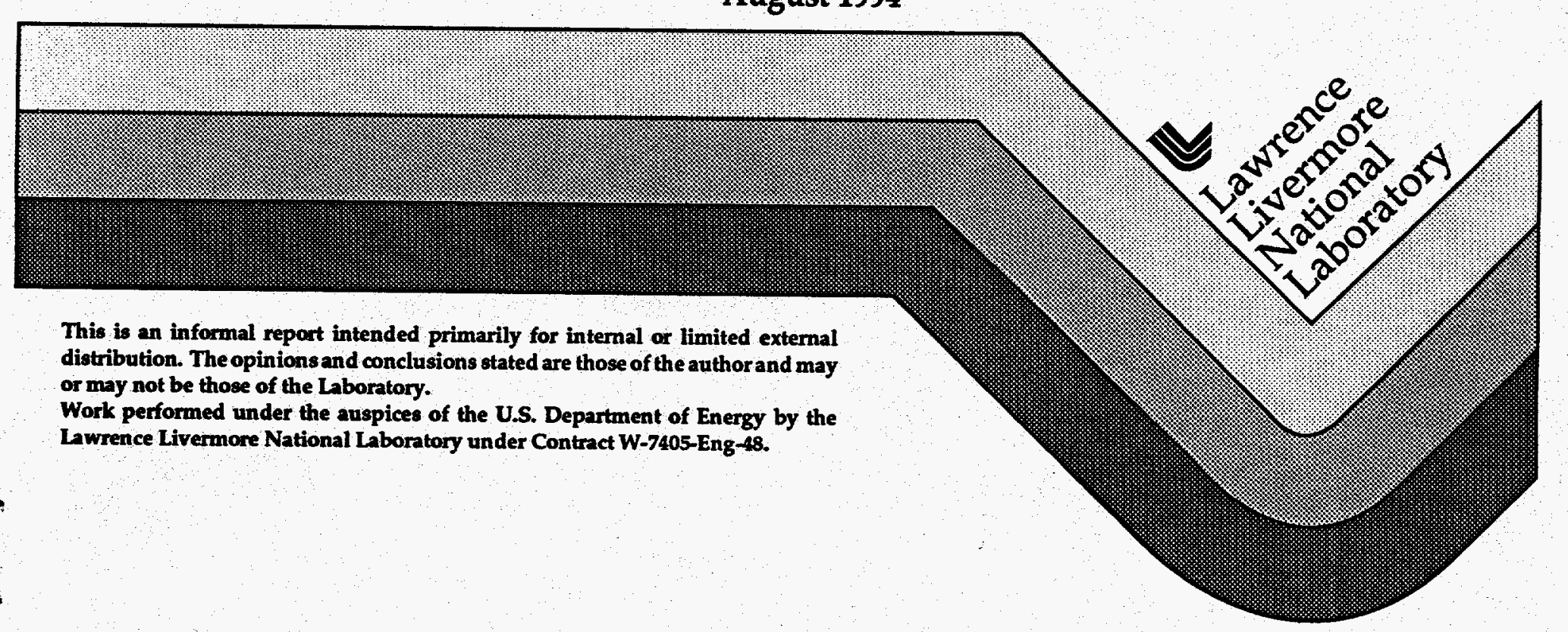


This document was prepared as an acccount of work sponsored by an agency of the United States Government. Neither the United States Government nor the University of California nor any of their employees, makes any warranty. express or implied, or assumes any legal liability or responsibility for the accuracy, completeness, or usefulness of any information, apparatus, product, or process disclosed, or represents that its use would not infringe privately own rights. Reference herein to any specific commercial products, process, or service by trade name, trademark, manufacturer, or otherwise, does not necessarily constitute or imply its endorsement, recommendation, or favoring by the United States Government or the University of California. The views and opinions of authors expressed herein do not necessarily state or reflect those of the United States Government or the University of California, and shall not be used for advertising or product endorsement purposes.

This report has been reproduced directly from the best available copy.

Available to DOE and DOE contractors from the Office of Scientific and Technical Information P.O. Box 62, Oak Ridge, TN 37831

Prices available from (615) 576-8401, FTS 626-8401

Available to the public from the National Technical Information Service

U.S. Department of Commerce 5285 Port Royal Rd., Springfield, VA 22161 


\section{DISCLAIMER}

Portions of this document may be illegible in electronic image products. Images are produced from the best available original document. 


\title{
BEGET: The B-Factory Event Generator Version 21
}

\author{
Douglas M. Wright \\ LLNL
}

\begin{abstract}
This note is a reference manual for the B-Factory Event Generator (BEGET V21) software package which generates physics events relevant to B-Factory detector studies. The package provides a standard framework that can easily interface to various external generators and simulation applications. Version 21 of BEGET contains a number of physics and background generators and is interfaced to the JETSET and KORALB generators and the GEANT and ASLUND simulation programs.
\end{abstract}

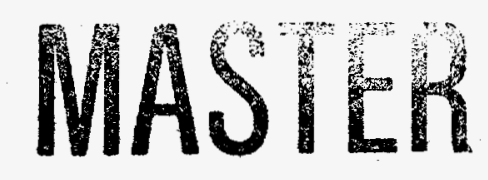

Questions and comments should be addressed to wright20@llnl.gov.

This document is also available in hypertext and postscript form: http://babar1.llnl.gov/wright/beget.html 


\section{Contents}

1 Introduction 1

$1.1 \Upsilon(4 \mathrm{~S})$ Decay . . . . . . . . . . . . . . . . . . . . . . . . . 2

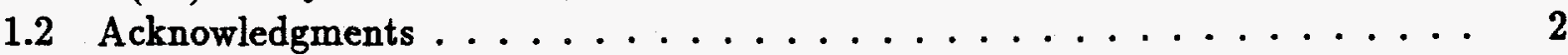

2 Using BEGET

2.1 Using BEGET for Generator Studies . . . . . . . . . . . . . . 3

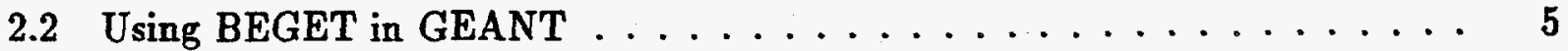

2.3 Using BEGET in ASLUND .................... 5

2.3.1 Comments on Particle Codes for ASLUND . . . . . . . . . . . . 6

2.4 User Hooks and Utilities . . . . . . . . . . . . . . . . . 7

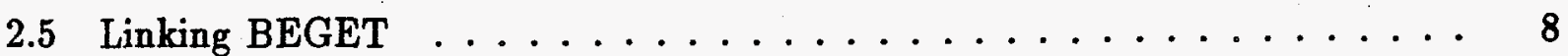

2.5.1 Selective Linking of Generators . . . . . . . . . . . 8

3 Installing BEGET $\quad 9$

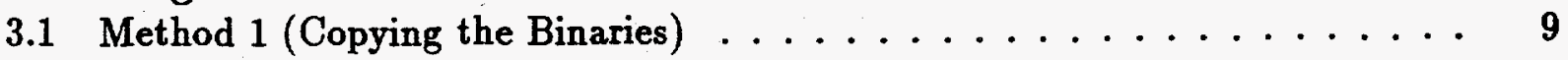

3.2 Method 2 and 3 (Compiling BEGET) ............... 9

3.3 Software Requirements . . . . . . . . . . . . . . . 10

4 Caveats $\quad 11$

5 Controlling BEGET $\quad 12$

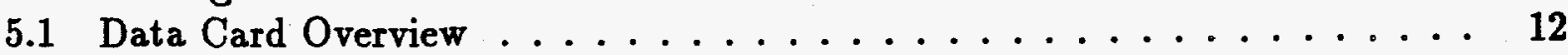

5.2 Data Card Default Values ...................... 13

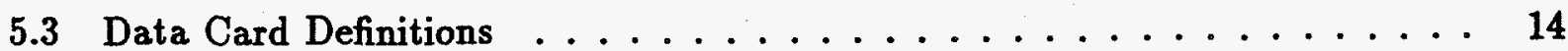

GEN: Choose what to generate .................. 14

BACK: Activate beam background from TURTLE files . . . . . . . . . 16

BACD: Directory to find TURTLE files . . . . . . . . . . . . . 17

CP: $\quad$ Select CP violation and/or $B^{0}-\bar{B}^{0}$ mixing . . . . . . . . . 17

B0B0: Selectively deactivate $B^{0}$ and/or $\bar{B}^{0}$ decay modes ........ 18

JPSI: $\quad$ Control $J / \psi$ decay modes . . . . . . . . . . . . . 19

JETD: Specify a file to read in JETSET decay information . . . . . . 19

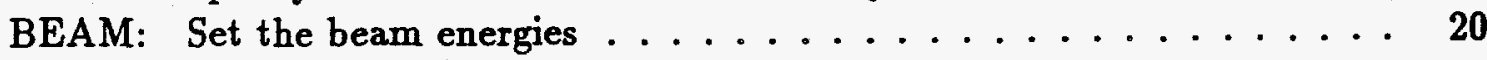

SBEAM: Set the beam energy spread .................. 20

VERT: Set the primary vertex location ................ 21

SVERT: Set the primary vertex spread ................ 21

IVERT: Set the primary vertex smearing method . . . . . . . . . . 21

GENP: Print event information on the terminal . . . . . . . . . . . 21

JETO: Print the decay table for the listed particles . . . . . . . . . . 22

RAN: Control the random number seeds .............. 22

EVNT: Set the number of events to generate . . . . . . . . . . . 23 
6 STDHEP Common Block

References 


\section{List of Tables}

1 Decay modes of interest for $\mathrm{CP}$ violation. . . . . . . . . . . 2

2 Particle Code Convention for $b \bar{b}$ resonances. . . . . . . . . . . 6

3 ASLUND version of LUCOMP mapping. . . . . . . . . . . . . 7

4 BEGET software requirements . . . . . . . . . . . . . . 10

5 STDHEP common block variables. . . . . . . . . . . . 24 


\section{Introduction}

The B-Factory Event Generator (BEGET) provides a simple framework for generating events appropriate for a detector at an asymmetric $e^{+} e^{-}$colliding beam detector. The current version of BEGET, Version 21, can be run in stand-alone mode (for generator level studies) or called from GEANT[1] or ASLUND[2].

BEGET has both built-in generators and interfaces to external generators, such as JETSET[3] and KORALB[4]. For all generators, BEGET handles global tasks such as setting beam energies, smearing the primary vertex, and boosting to the final (beam asymmetric) reference frame. It also has a number of features to facilitate CP asymmetry studies. For example, there are switches to activate the rare $B$ decay modes to $\mathrm{CP}$ eigenstates and routines that simulate $\mathrm{CP}$ violation and mixing.

Calling BEGET produces a single event stored in a FORTRAN common block in a format defined by the STDHEP software package[5]. The common block contains a list of the generated particles with their associated four-vectors and vertices. STDHEP defines not only the common block but also the particle code convention, so that the interface to any external simulation package is handled by a single subroutine that reads the common block.

BEGET is controlled by an ASCII file containing keywords and parameters, called 'data cards.' The data cards are defined and read in with the FFREAD[6] package from the CERN library. Except for some of the beam related background generators, no external data files are required to run the program. All defaults are built-in; however, the user can override nearly every aspect of the program through data cards and external files. For example, the decay table for selected particles (or all particles) can be replaced.

The generators currently available in BEGET are:

1. Single particle. The user specifies the particle and four-vector range.

2. $\Upsilon(4 S)$ decay via JETSET.

3. Hadronic continuum events from JETSET (i.e. $e^{+} e^{-} \rightarrow q \bar{q}$ ).

4. Quark "Onia" resonance decays via JETSET.

5. KORALB $\tau^{+} \tau^{-}$decays.

6. Beam background from off-momentum particles.

7. Background from beam-gas interactions.

8. Cosmic rays.

Additional generators and decay packages (e.g. QQ from CLEO) are in the works, and users are encouraged to interface their own favorite generators. 


\section{$1.1 \Upsilon(4 S)$ Decay}

The current $\Upsilon(4 S)$ generator uses JETSET and is a modified version of what is found in the native ASLUND generator. A proper treatment of $\Upsilon(4 S)$ production that includes the upsilon width has not yet been implemented. The $\Upsilon(4 S)$ decays into an equal mixture of $B^{0} \bar{B}^{0}$ and $B^{+} B^{-}$final states.

The standard JETSET decay tables are modified with updated (circa 1992) heavy meson decay data $(B$ and $D$ ) from CLEO and ARGUS. See subroutines BEGFILCST and BDECAY for more details. I have added the $B^{0}$ decay modes of interest for CP violation studies which are listed in Table $1^{1}$

Table 1: Decay modes of interest for CP violation.

\begin{tabular}{|l|r|}
\hline$B^{0}$ Decay Mode & BR $\left(\times 10^{-4}\right)$ \\
\hline$\psi K_{L}^{0}$ & 3.85 \\
$\psi K_{S}^{0}$ & 3.85 \\
$\psi K^{* 0}$ & 12.60 \\
$D^{+} D^{-}$ & 6.00 \\
$D^{*+} D^{*-}$ & 15.00 \\
$\pi^{+} \pi^{-}$ & 0.20 \\
$\rho^{ \pm} \pi^{\mp}$ & 0.80 \\
$a_{1}^{ \pm} \pi^{\mp}$ & 0.80 \\
\hline
\end{tabular}

A built-in switch allows all $B^{0}$ (or $\bar{B}^{0}$ ) decay modes to be turned off except for a selected mode. For example, to generate the following decay:

$$
\Upsilon(4 \mathrm{~S}) \rightarrow\left(B^{0} \rightarrow \psi K_{L}^{0}\right)\left(\bar{B}^{0} \rightarrow \mu^{-} \bar{\nu}_{\mu} X\right)
$$

One would use the following data card: Вово 11 3. The keyword is Вово, the first number specifies the $B^{0}$ decay $\left(11=\psi K_{L}^{0}\right)$ and the second specifies the $\bar{B}^{0}$ decay $\left(3=\mu^{-} \bar{\nu}_{\mu} X\right)$. Instead of using the built-in switches the user can alter the $B^{0}$ decay, or any other decay, with an external file that contains the desired changes to the decay table (data card JETD).

By default, CP violation and $B^{0}$ mixing is turned off. The user can activate it with data card CP. In the current implementation, the CP part only works on pure CP eigenstate modes. There is a also an option in JETSET to do $B^{\mathbf{0}}$ mixing. Currently, the user must activate this by hand (i.e. by setting the value in the appropriate JETSET common block).

Section 5 on page 12 contains a complete description of the available data cards.

\subsection{Acknowledgments}

Acknowledgments traditionally appear at the end of a document but are included here so that they do not become lost to the reader among the details of the text.

\footnotetext{
${ }^{1}$ The branching ratios match those in the BaBar LOI [7]
} 
BEGET began as a synthesis of old and new subroutines. The old was taken primarily from ASLUND and the only credit that $I$ can ascribe comes from comments left in the source code itself. The ASLUND simulation began as a modification of another fast, parametrized simulation. Alan Weinstein created the original ASLUND and Art Snyder maintains and develops the current version. Some of the JETSET modifications and utilities should be attributed to Patrick Roudeau and Jon Guy. In particular the heavy meson decay modifications come from them. The routines that simulate CP asymmetry are due to Alan Weinstein with modifications from Gautier Hamel de Monchénault, Dave Coupal, and Walt Innes.

A number of people have contributed to BEGET since its first release (Version 0, January 1994). In particular Fred Kral has implemented the KORALB interface, the beam-gas interaction interface, and a cosmic ray generator. Ezio Torassa contributed a routine for picking background rays from TURTLE files prepared by Art Snyder. Dave Coupal has made extensive changes to this routine.

Contributions from others are soon to be incorporated and as the program matures I hope that this section continues to grow.

\section{Using BEGET}

A single event is generated by calling subroutine BEGET (i.e. CALL BEGET). This fills the STDHEP common block with the final state particles and the intermediate particles that decayed. Subsequent calls to the BEGET subroutine put a new event into the common block.

The general purpose part of BEGET must be initialized before the first call to the BEGET subroutine. This initialization is handled by a different subroutine that depends on whether one is running BEGET stand-alone or from GEANT or ASLUND. The main purpose of the initialization is to read in the data cards that define what to generate.

The proper calling structure is already built-in to ASLUND and BBSIM, ${ }^{2}$ so users of those programs do not need to modify their code. The initialization for a particular generator package (e.g. JETSET) is aufomatically called in the first pass through the BEGET subroutine, so no special user action is required. Be aware that BEGET is not currently configured to allow input parameters to change between generated events.

\subsection{Using BEGET for Generator Studies}

The following two program examples can be found in the example directory of the source code distribution. Shell scripts beget 1 and beget 2 contain the proper compilation and linking commands.

To make a stand-alone version of BEGET, use a main program like the following (which generates 10 events). ${ }^{3}$

program main

call beginput ! define and read in data cards

${ }^{2}$ BBSIM = B-Factory GEANT detector simulation.

${ }^{3}$ Unlike BBSIM, users do not need to copy any of the BEGET source code. 
do $n=1,10$

call beget ! generate an event

enddo

end

Link the program using the following command,

$$
\text { f77bb - link -o beget1.exe beget1.f '\$BFLIB/Iibbeget.Link" }
$$

where the main program has been saved in file beget 1. $f$. The back quotes (') are important, they cause the output of the libbeget.Link script to be included on the command line. The libbeget. Link script produces the appropriate list of libraries needed to link BEGET. Linking is discussed in detail in Section 2.5.

This small example results in a complete, functioning version of BEGET. It can be used to familiarize oneself with the function of the various BEGET data cards.

For generator studies one needs access to the STDHEP common block, which is defined in the include file stdhep.inc. ${ }^{4}$ A slightly more complicated main program that illustrates how one might read the common blocks is given below.

program main

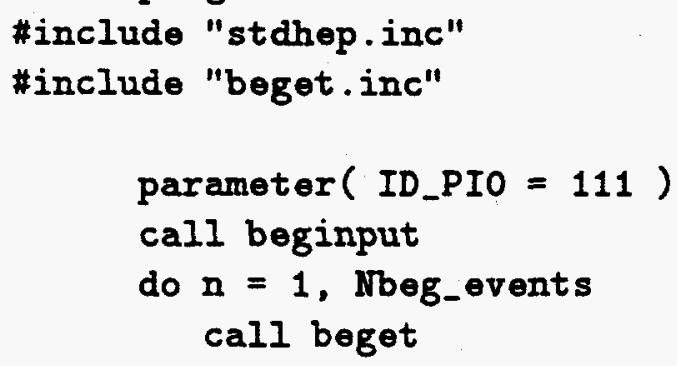

This example merely counts the number of $\pi^{0}$ 's in the current event by looping over all of the particles and checking the particle code in array IDHEP. Section 6 contains a summary of all of the variables defined in the STDHEP common block.

The BEGET internal common block, defined in beget.inc, gives the user access to the values of the input data cards and other internal BEGET variables. To compile and link this program, one must specify the location of the includes files. The following commands will do the trick:

\footnotetext{
${ }^{4}$ The include file is stored with the source code which is typically kept in \$BFROOT/src/stdhep/pro.
} 
f77bb -cPp "-I\$BFROOT/src/beget/pro/src -I\$BFROOT/src/stdhep/pro" beget2.F

f77bb -link -o beget2.exe beget2.o '\$BFLIB/libbeget.Link"

The above examples expect that the $f 77 \mathrm{bb}$ shell script is used for the compilation and linking of the program, however, this is not a requirement. The libbeget.Link script is general enough to work with any UNIX linker and the main program will work with a generic FORTRAN compiler if the $\mathrm{C}$ style includes (\#include) are changed to FORTRAN style includes.

\subsection{Using BEGET in GEANT}

To use BEGET with GEANT ${ }^{5}$ make a GUKINE subroutine like the following.

subroutine gukine

call beget_geant ! generate an event

end

and make sure that the following call is made before the call to GFFGO.

call begffkey ! define BEGET FFREAD data cards

The GEANT implementation works by calling the BEGET subroutine and then subroutine HEP2GEANT to load the contents of the STDHEP common block into the GEANT KINE and VERT banks.

Link your GEANT program with '\$BFLIB/libbeget.Link` as an argument to your link command (note the 's are important). For more information on linking see Section 2.5.

\subsection{Using BEGET in ASLUND}

The BEGET interface is already built in to ASLUND, however, it is not linked into the ASLUND executable by default. To link with BEGET you must uncomment the line

\section{\#define BEGET}

in the ASLUND user/Imakefile and remake your executable.

Once you have a version of ASLUND that contains BEGET, set IFL $=-99$ in the runtime input file, this turns off the built-in generator and turns on BEGET. BEGET data cards should be embedded in the runtime file, they are not read in separately. For ASLUND to recognize the BEGET data cards ; BEGETBEGIN must appear on a separate line before the first card and ;BEGETEND must follow on a separate line after the last card, For example:

\section{;BEGETBEGIN}

BOBO 311

GENP 1

;BEGETEND

${ }^{5}$ BBSIM users: BEGET is already implemented in BBSIM, so there is no need to change any source code. 
This only applies to ASLUND. The stand-alone and GEANT version of BEGET do not require ;BEGETBEGIN, ;BEGETEND.

The default beam energies are taken from ASLUND variables instead of BEGET, but the defaults can be overridden by the BEGET data cards BEAM and SBEAM. Also, ASLUND does its own vertex smearing so data cards VERT, SVERT, IVERT should not be used at this time.

ASLUND is not built around STDHEP, it looks directly at the JETSET common blocks to find the generated particles. The BEGET interface to ASLUND makes use of a STDHEP translation routine that converts STDHEP to JETSET. So non-JETSET generators (in BEGET) are automatically available in ASLUND.

\subsubsection{Comments on Particle Codes for ASLUND}

Some ASLUND extensions to the JETSET particle code conventions are not compatible with STDHEP (i.e. particle codes are used that either map incorrectly into the STDHEP scheme or do not exist at all). In general this is not a problem. Users who modify the JETSET decay tables with an external file may run into trouble if they try to use the same file with BEGET. However, by following a few guidelines an external file can be made that works with both BEGET and the ASLUND native generator.

Traditionally ASLUND uses 40553 as the code for $\Upsilon(4 S)$, which is not defined by JETSET, but is defined by STDHEP as code 70553. The STDHEP convention for $b \bar{b}$ resonances is given in Table 2. The ASLUND user may select particle 70553 as $\Upsilon(4 S)$ but must be

Table 2: Particle Code Convention for $b \bar{b}$ resonances.

\begin{tabular}{|l|c|c|}
\hline & \multicolumn{2}{|c|}{ Particle Code } \\
\cline { 2 - 3 } & ASLUND & STDHEP \\
\hline$h_{b}(2 P)$ & & 40553 \\
$\chi_{b 1}(2 P)$ & & 50553 \\
$\Upsilon(3 S)$ & & 60553 \\
$\Upsilon(4 S)$ & 40553 & 70553 \\
$\Upsilon(5 S)$ & 50553 & 80553 \\
\hline
\end{tabular}

careful to use the correct "compressed" code associated with it. Particle codes in JETSET are "compressed", i.e. mapped to a contiguous range of numbers 1-500. The function LUCOMP ( $k f$ ) returns the compressed code for particle code kf. JETSET does not define the mapping for particles with compressed codes $400-500$. It is up to the user to define the mapping.

The ASLUND version of LUCOMP has a mapping given in Table reflucomp. In ASLUND there are (at least) two ways to get a compressed code of 404 . For example 70553 and 4000511 both compress to 404 . To make decay table files that are compatible with both the native ASLUND generator and BEGET, use code 70553 and compressed code 404 for $\Upsilon(4 S)$, and make sure that any other user particles give a compressed code greater than 410 . 
Table 3: ASLUND version of LUCOMP mapping.

\begin{tabular}{|r|r|}
\hline KF & LUCOMP(KF) \\
\hline 40553 & 401 \\
50553 & 402 \\
60553 & 403 \\
70553 & 404 \\
80553 & 405 \\
$\mathrm{nXXXXXX}$ & $400+$ abs(n) \\
\hline
\end{tabular}

The stand-alone and GEANT version of BEGET do not respect the LUCOMP mapping of ASLUND for particle codes $n \times x \times x \times x$ (i.e. $>99,999$ ) because of the possible inconsistencies stated above. So decay table files can be shared between the ASLUND native generator and BEGET only within ASLUND. With sufficient user interest, the discrepancies in the LUCOMP mapping could be eliminated.

\subsection{User Hooks and Utilities}

A few hooks are provided to allow a more detailed control of BEGET. The following subroutines are dummies in the BEGET library. The user may write his/her own subroutines and link them before the BEGET libraries.

- Subroutine BEGUSER is called instead of any other generator, if GEN -99. It provides a means for users to write their own generators or develop a new interface to an external generator.

- Subroutine BEGUNIT is called after the requested generator has been initialized, but before any events are generated, to give the user an opportunity to change the initialization of the generator.

- Logical Function BEGUREJECT allows the user to reject the event and request a new one before returning from the BEGET subroutine.

None of the subroutines or functions take any arguments.

Some BEGET subroutines that may be of interest to users are:

- HEPROBO performs Lorentz boosts on all of the particles in the STDHEP common block.

- BEGHBOOK initializes the HBOOK histogramming package.

- HEP2GEANT reads the STDHEP common block and places the undecayed particles in the GEANT KINE and VERT banks.

Please refer to the source files in the src directory of the BEGET distribution for more information. 


\subsection{Linking BEGET}

Since a number of libraries and object modules are needed to link BEGET with your program a shell script (libbeget.Link) is provided to produce the proper linker arguments. This not only simplifies linking but also allows the easy switching of BEGET versions and the selective linking of external generators (such as KORALB).

The script produces a text string that contains the actual linker arguments in the appropriate order. The standard unix trick of using ' permits the output of the script to be included as part of another command, e.g. $177 \mathrm{bb}-$ link test.o 'libbeget. Link". To see the output of the script enter it as a regular UNIX command without the 's.

The BEGET version number is hardwired into the script so that it always links a specific version (even if the script is accessed via a link). The script looks for the beget libraries in the same directory in which the script resides (e.g. \$BFLIB/libbeget.Link looks in \$BFLIB). In addition the script requires two environment variables \$BFLIB and \$CERN_ROOT, the first is needed to locate the STDHEP library and the second is used to locate the CERN libraries.

A few examples:

-\$BFLIB/libbeget.Link'

'\$BFLIB/libbeget.v021.Link"

'\$HOME/libbeget.v999.Link'
Link the defaults version of BEGET.

Link BEGET version 21.

Link BEGET version 999 found in

the user's home directory.

\subsubsection{Selective Linking of Generators}

By default BEGET links the JETSET library. JETSET is required for the random track generators (to do the particle decays if the user requested a non-stable particle) and the $\Upsilon(4 \mathrm{~S}), q \bar{q}$, and quark "Onium" generators. Other external generators are not linked automatically. This keeps the resulting executable small, minimizes linking time and eliminates the need for every site to install every generator.

Currently, the only external generator available is KORALB. To activate it you must install the KORALB library on your system (a development version is already installed at SLAC) and add an argument to the linker script to tell BEGET where it is.

For example if KORALB is installed as libkoralb.vdev .a in the \$BFLIB directory then

$$
\text { `\$(BFLIB)/libbeget.Link - Ikoralb.vdev" }
$$

would produce a BEGET program that can access KORALB. ASLUND users must edit a line in the ASLUND user/Imakefile to look like the following:

$$
\text { BEGETLIBS }=-\$(B F L I B) / \text { libbeget.Link aslund -lkoralb.vdev` }
$$

To actually generate KORALB events the user must select the GEN 10 data card at run time.

New external generators (for example $Q Q$ ) will be made available via the same mechanism. They will not be linked automatically unless the user requests them. 


\section{Installing BEGET}

The master copy of BEGET is stored on the SLAC unix cluster in CVS. There are three method to install it at a remote site:

1. Copy the binaries and include files.

2. Copy the 'tar' file and compile BEGET.

3. Check out the CVS version and compile BEGET.

The third method has the advantage that bug fixes can be automatically incorporated at the remote site.

BEGET has been successfully installed under the following operating systems: HP-UX, AIX, SunOS, Alpha/OSF, and Ultrix. Any problems with these or other platforms should be reported to wright20eIlnl.gov.

\subsection{Method 1 (Copying the Binaries)}

Copy the most recent version of the following files from a site that has installed BEGET, of course the CPU type must match your system. ${ }^{6}$

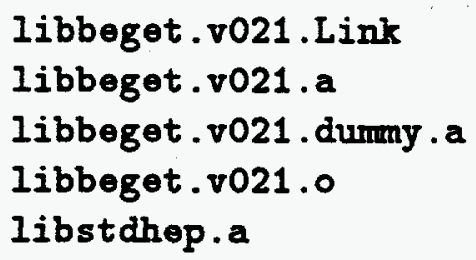

Make a link libbeget.Link to libbeget.v021.Link.

If you wish to perform generator studies you must also copy two include files:

\$BFROOT/src/beget/v021/beget.inc

$\$$ BFROOT/src/stdhep/pro/stdhep.inc

\subsection{Method 2 and 3 (Compiling BEGET)}

Installing BEGET from the source code is a three step process:

1. Copy the source code to your system from either

- the most recent tar file in \$BFROOT/src/beget

- via CVS: cvs -R co -r v021 -d v021 beget

Store the source code in \$BFROOT/src/beget/v021 and maintain a pro link to the current version, which in this case is $\mathrm{v} 021$.

\footnotetext{
${ }^{6}$ The SLAC unix cluster has the IBM RS6000 version (\$BFROOT/lib.aix6000) and the HP version (\$BFROOT/lib.hp).
} 
2. Compile the code. Type gmake in the BEGET directory you just created. This builds the libraries in the current directory. You may have to edit the GNUmakefile to specify the location of the stdhep include file, i.e. edit the line:

hepinc :=\$(BFROOT)/src/stdhep/pro

3. Move the libraries to the $\$ B F L I B$ area. Type gmake install to move the libraries from the working directory to the $\$ B F L I B$ area.

\subsection{Software Requirements}

Table 4 outlines the software required to install BEGET which does not come with the BEGET distribution.

Table 4: BEGET software requirements

Required Environment Variables:

BFLIB

final location of BEGET libraries

CERN_ROOT

location of cern libraries (e.g. /cern/pro)

Required to install BEGET:

$\mathrm{f} 77 \mathrm{bb}$

libstdhep.a

stdhep.inc

libpacklib.a

libmathlib.a

libjetset.a

Optional

libkoralb.a shell script for compiling FORTRAN

STDHEP library include file for STDHEP common block

CERN library

CERN library

CERN library

KORALB library 


\section{Caveats}

Caveats on running BEGET.

- When linked to GEANT the following particles: $K_{S}^{0}, \Sigma^{ \pm 0}, \Xi^{-0}, \Lambda^{0}, \Omega^{-}$, are declared to be stable (which JETSET normally decays). This allows them to be passed on to GEANT which then handles their decay.

- The $\Upsilon(4 S)$ width is not used when beam smearing is activated.

- KORALB does not handle smearing of beam energies. This would require reinitializing KORALB at every event, which has not been implemented.

- When linked to ASLUND, the default beam energies are taken from ASLUND variables instead of BEGET, but the defaults can be overridden by the BEGET data cards BEAM and SBEAM.

- When running ASLUND do not use data cards VERT, SVERT, or IVERT, since ASLUND does its own vertex smearing after the event has been generated.

- When linked to ASLUND, BEGET uses the ASLUND version of subroutine LUCOMP instead of its own version. The mapping of user defined particles is different in the two cases. Power users who exploit the mapping of these non-standard particles should review Section 2.3.1.

Carefully constructed decay table files can therefore be used with the ASLUND native generator or BEGET. The stand-alone and GEANT version of BEGET use a different version of LUCOMP which is more robust. With sufficient user interest, the discrepancies in the LUCOMP mapping could be eliminated. 


\section{Controlling BEGET}

This section describes the data cards used to control BEGET. The data cards are read in with the FFREAD package from the CERN library. The defaults are set in subroutine BEGFFKEY and BEGINIT.

\subsection{Data Card Overview}

\begin{tabular}{|l|l|}
\hline Data Card & Function \\
\hline \multicolumn{2}{|c|}{ Generator Controls } \\
\hline GEN & Choose what to generate \\
BACK & Activate beam background from TURTLE files \\
CP & Specify a directory containing TURTLE files \\
\hline \multicolumn{2}{|c|}{ Select CP violation and/or $B^{0}-\bar{B}^{0}$ mixing } \\
\hline BOB0 & Selectively deactivate $B^{0}$ and/or $\bar{B}^{0}$ decay modes \\
JPSI & Control J/ $\psi$ decay \\
JETD & Specify a file to read in JETSET decay information \\
\hline \multicolumn{2}{|c|}{ Beam Controls } \\
\hline BEAM & Set the beam energies \\
SBEAM & Set the beam energy spread \\
VERT & Set the primary vertex location \\
SVERT & Set the primary vertex spread \\
IVERT & Set the primary vertex smearing method \\
\hline \multicolumn{2}{|c|}{ General Controls } \\
\hline GENP & Print event information on the terminal \\
JETO & Print the decay table for the listed particles \\
RAN & Control the random number seeds \\
EVNT & Set the number of events to generate \\
REJE & Control user event rejection \\
\hline
\end{tabular}




\subsection{Data Card Default Values}

\begin{tabular}{|c|c|c|}
\hline Data Card & $\begin{array}{l}\text { Default } \\
\text { Value }\end{array}$ & Default Function \\
\hline \multicolumn{3}{|r|}{ Generator Controls } \\
\hline GEN & 2 & Generate $\Upsilon(4 \mathrm{~S}) \rightarrow \bar{B}^{0} \bar{B}^{0}$, decay using JETSET. \\
\hline BACK & 0 & do not add TURTLE background \\
\hline BACD & $"$ & default directory to find TURTLE files is \\
\hline q & & \$BFROOT/src/beget/pro/dat \\
\hline CP & 0 & No $C P$ violation or $B^{0}-\bar{B}^{0}$ mixing. \\
\hline \multicolumn{3}{|r|}{ Decay Controls } \\
\hline B0B0 & 11 & Turn on all $B^{0}$ decays. \\
\hline JPSI & -1 & Turn on all $J / \psi$ decays, unless BOBO is not 11 (see below) \\
\hline JETD & $"$ & Do not read any external decay file \\
\hline \multicolumn{3}{|r|}{ Beam Controls } \\
\hline$\overline{\mathrm{BEAM}}$ & $9.0-3.109$ & $p_{z}\left(e^{-}\right)=9.0 \mathrm{GeV} / \mathrm{c}, p_{z}\left(e^{+}\right)=-3.109 \mathrm{GeV} / \mathrm{c}$ \\
\hline SBEAM & 0.0 . & zero energy spread on beam (no smearing) \\
\hline VERT & 0.0 .0 . & event origin is $(0,0,0)$ \\
\hline SVERT & 0. 0.0 . & no vertex smearing \\
\hline IVERT & 000 & use gaussians (sigmas set by SVERT) \\
\hline \multicolumn{3}{|r|}{ General Controls } \\
\hline GENP & 00 & Do not print the events on the terminal. \\
\hline JETO & $0 * 20$ & no print out of decay tables \\
\hline RAN & 10 & use default seed $\# 1=987654321$ \\
\hline EVNT & 1 & set Mbeg_events $=1$ \\
\hline REJE & $\mathbf{0}$ & accept all events \\
\hline
\end{tabular}




\subsection{Data Card Definitions}

GEN: Choose what to generate

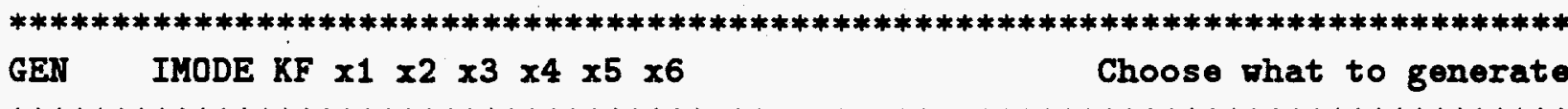

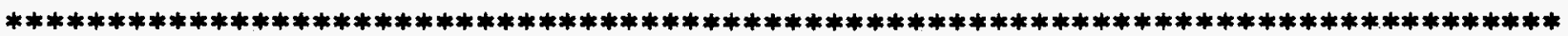

GEN
200.0 .0 .0 .0 .0$.
Defauls

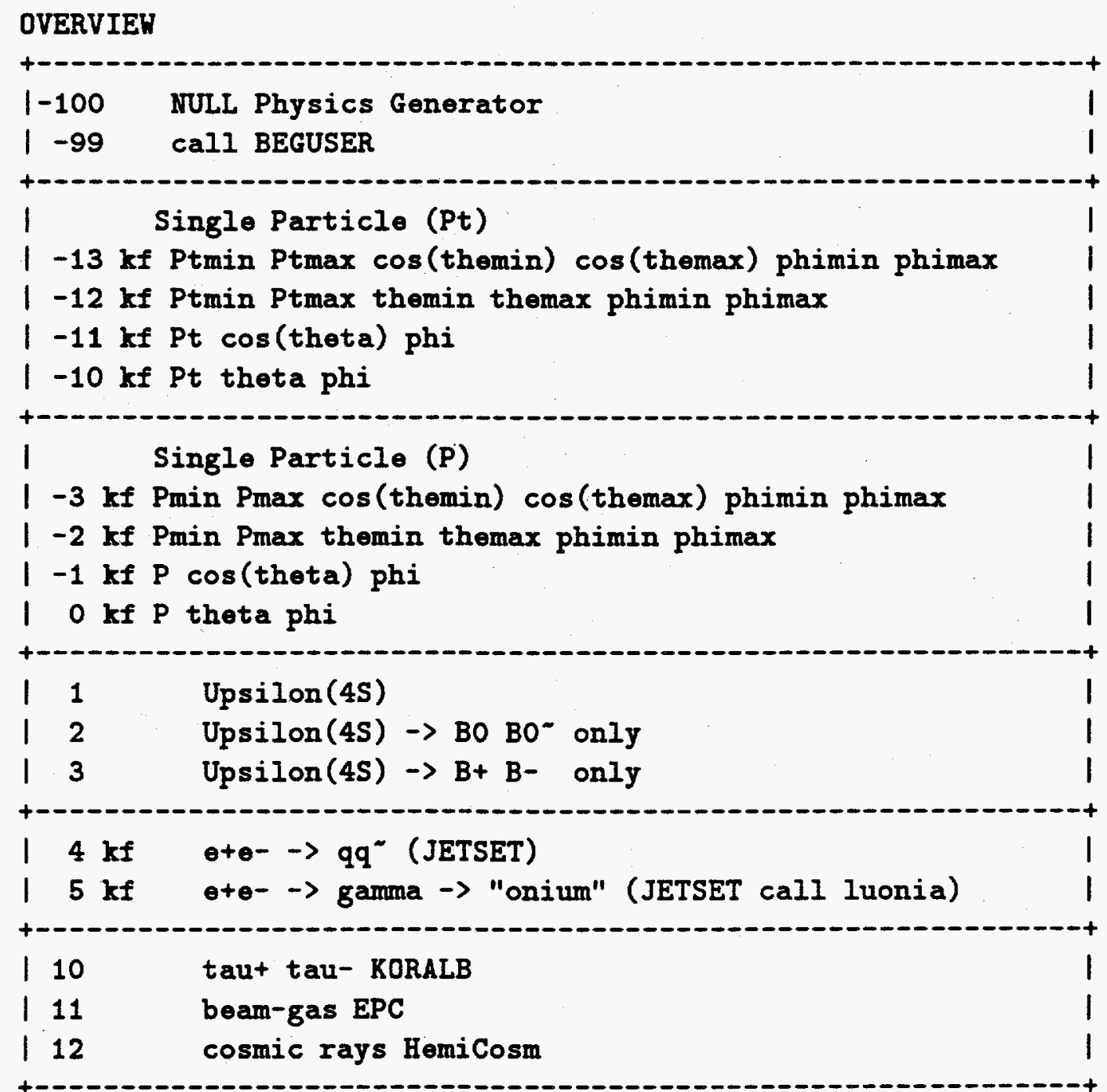

DETAILS

IMODE=0 User defined particle and four-vector. The particle is 
allowed to decay using JETSET.

$K F=$ JETSET code to identify particle

$\times 1 \times 2 \times 3=P$ Theta Phi

$\times 4 \times 5 \times 6=$ ignored

$P$ = momentum of particle in lab frame (GeV/c)

Theta, Phi = direction of mom. vector in lab frame (degrees)

IMODE $=-1$ same as IMODE $=0$ except :

$\times 1 \times 2 \times 3=p \cos ($ theta) $\mathrm{Phi}$

Angles in degrees, Momentum in GeV/c

IMODE $=-2$ same as IMODE=0 except :

$x 1 \times 2=$ Pmin Pmax

x3 $\times 4=$ The_min The_max

$x 5 \times 6=$ Phi_min Phi_max

A random value for $P$, theta and $p h i$ is chosen for each event in the ranges specified by $\times 1-x 6$

Angles in degrees, momentum in $\mathrm{GeV} / \mathrm{C}$

IMODE $=-3$ same as IMODE $=-2$ except :

$x 3 \times 4=\cos ($ The_min $) \cos ($ The_max $)$

Angles in degrees, momentum in $\mathrm{GeV} / \mathrm{C}$

IMODE $=-10$ to -13 same as IMODE 0 to -3 except :

Pt (momentum transverse to beam pipe) instead of $P$ momentum in $\mathrm{GeV} / \mathrm{C}$

IMODE=-99 Call subroutine BEGUSER to generate the event.

IMODE $=-100$ Do not call any physics generators.

IMODE=1 Generate Upsilon(4S) at rest in cm frame. Decay using JETSET and boost to lab frame.

IMODE $=2$ Same as IMODE=1 with Upsilon(4S) $\rightarrow$ BO BO decay only. 


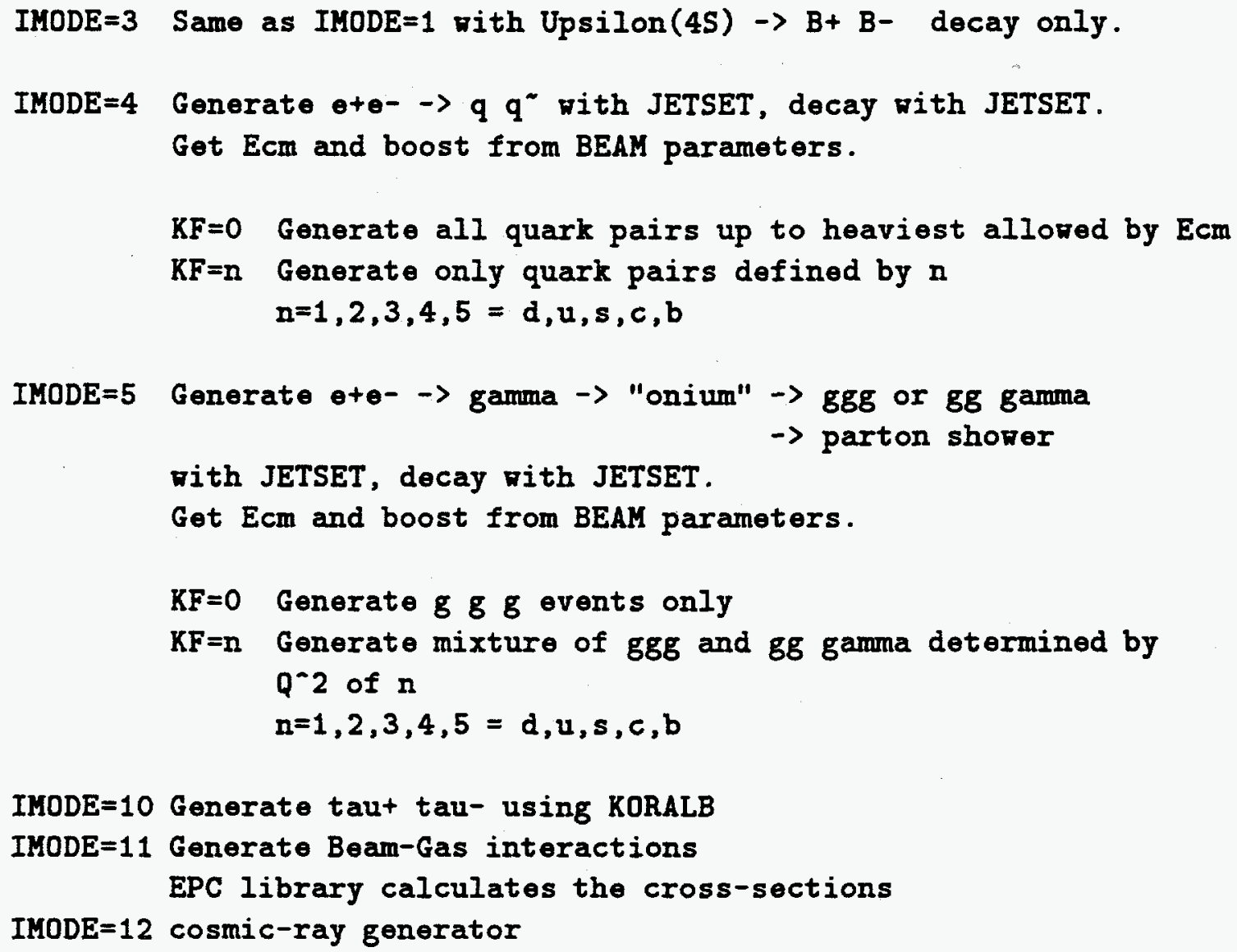

BACK: Activate beam background from TURTLE files

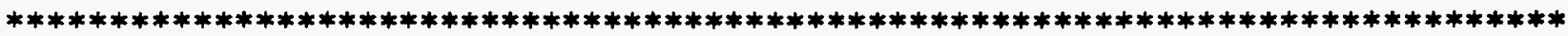
BACK Iback Tback 'file' Activate beam background from TURTLE files **************************************************************************** BACK 0 3."' Defaults

Add off-momentum beam particles and bremsstrahlung photons to the current event. The current implementation samples particles from a set of TURTLE files previously prepared.

If no GEN data card is present then no physics generator will be called, i.e. the event will contain only background.

Iback

- No background is added

1 Generate background by picking particles out of 


\section{previously prepared TURTLE files \\ 2 Read background from a file previously prepared from Iback=1 (the filename is required, see the examples belor) \\ Tback = Amount of time (in microseconds) to accumulate background file Save the generated background particles in a file (Iback=1)}

examples :

BACK 1 \# generate background for 3 micro seconds per event BACK 14 . \# generate background for 4 micro seconds per event

BACK 1 4. 'back.out' \# same as above and save the selected background

BACK 2 'back.out' \# read the particles from file back.out

\# particles in back.out

\# (one event at a time)

BACD: Directory to find TURTLE files

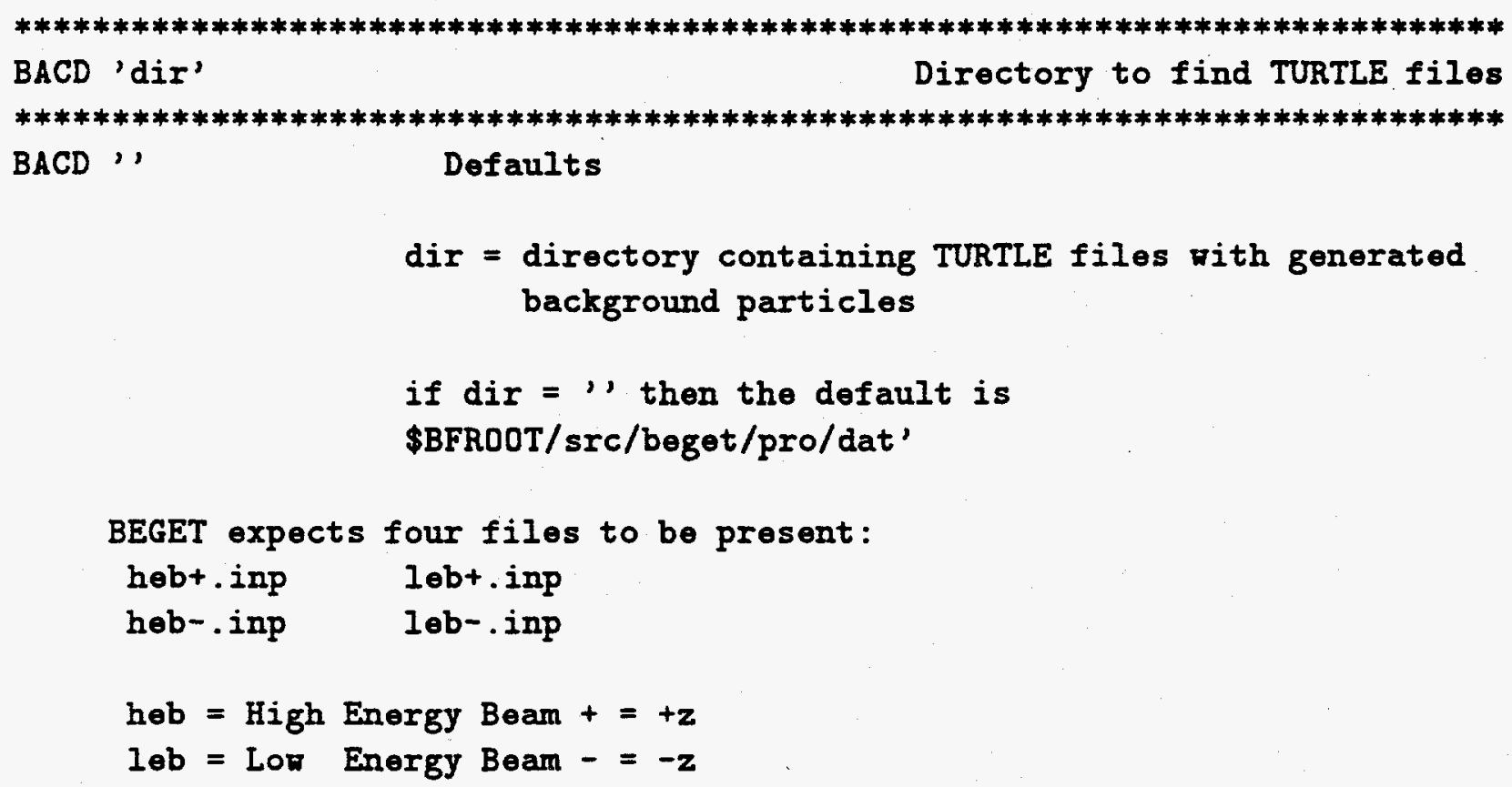

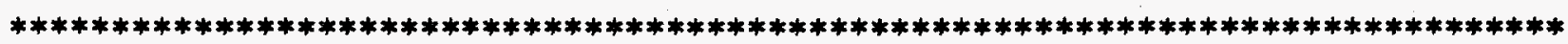
BACD ', Defaults background particles

CP: Select CP violation and/or $B^{0}-\bar{B}^{0}$ mixing 


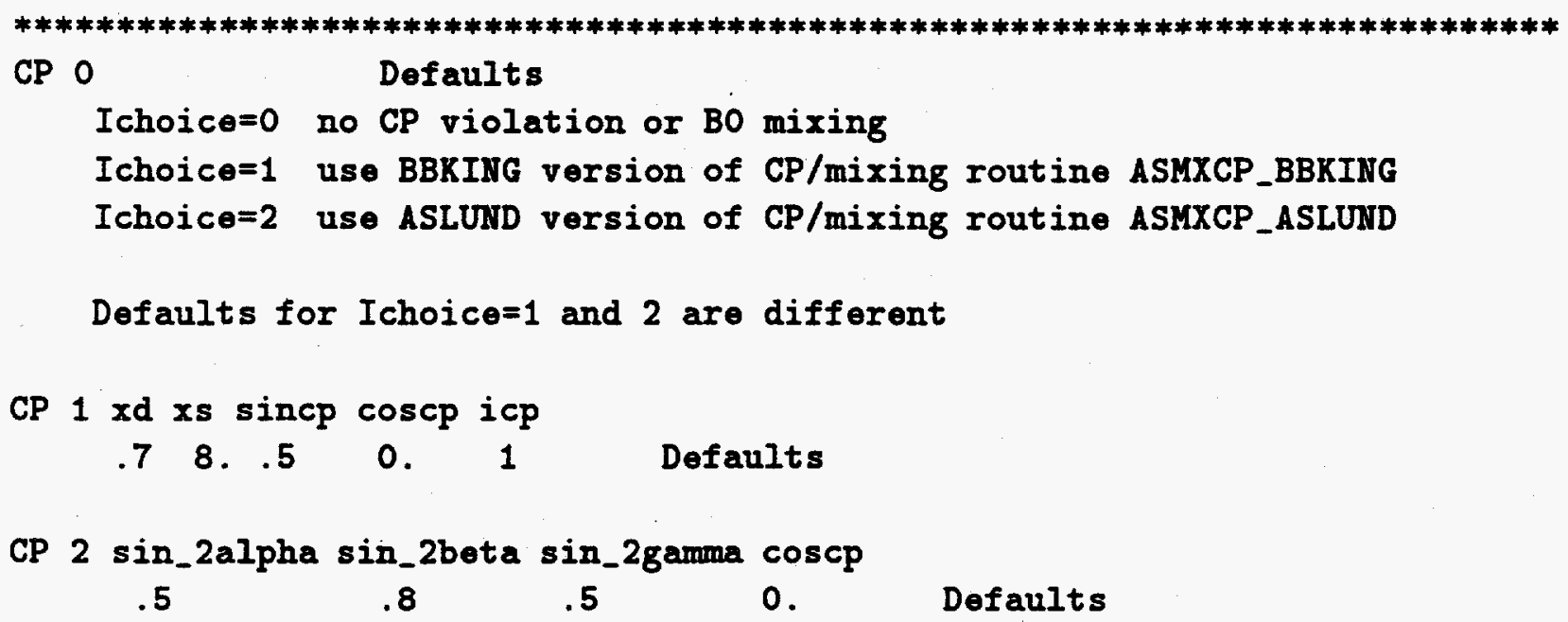

B0B0: Selectively deactivate $B^{0}$ and/or $\bar{B}^{0}$ decay modes

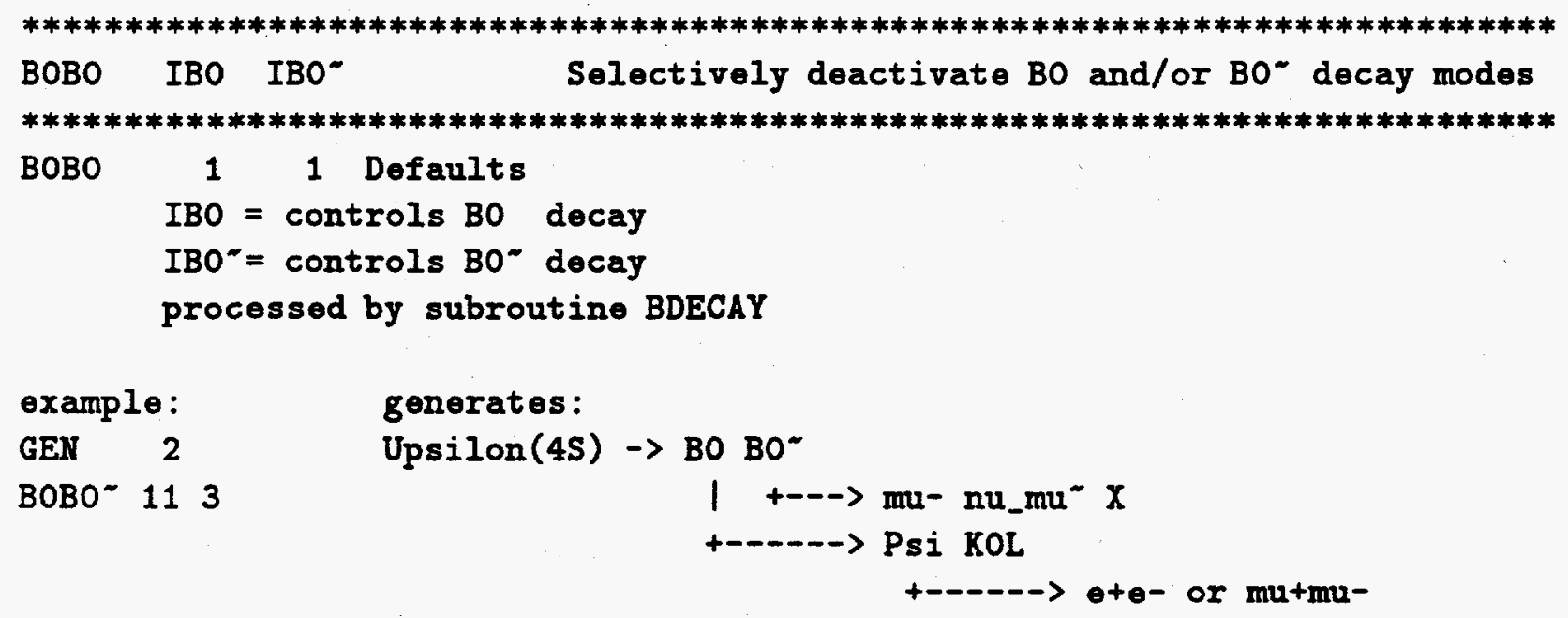

For decay modes 11,12, and 13 the Psi decay modes are sritched to ete- and mu+mu- only. The user can override this vith

the JPSI data card (see below)

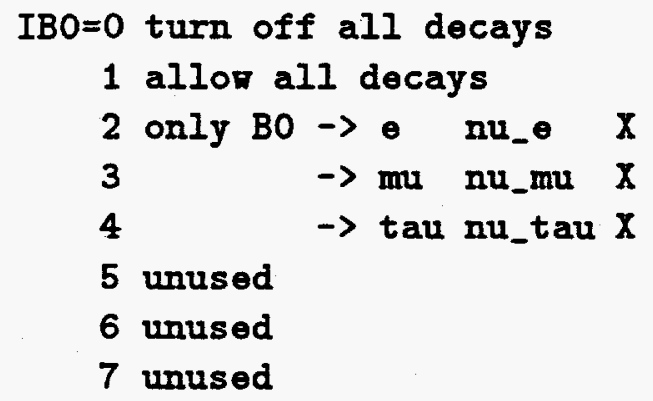




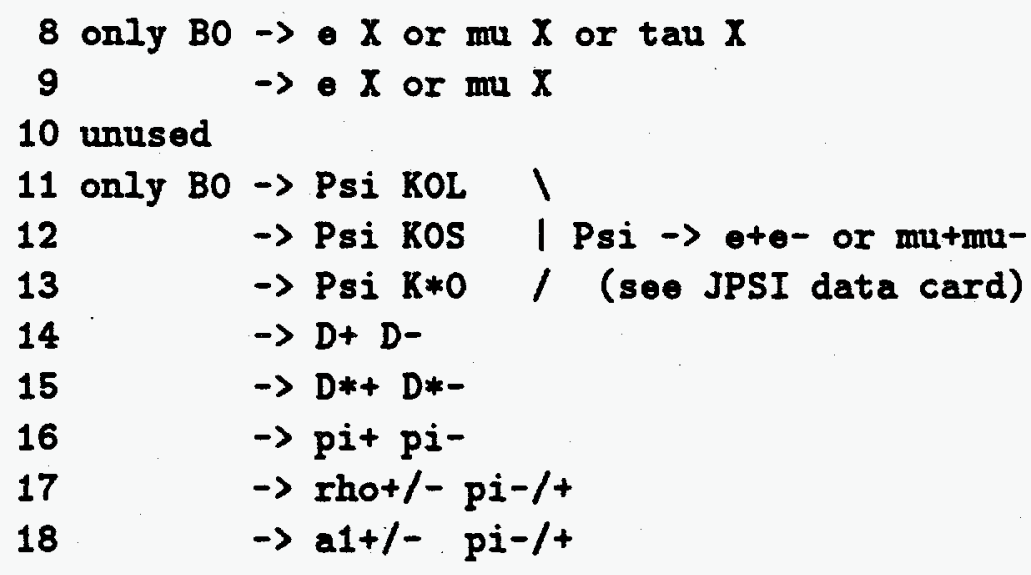

JPSI: Control $J / \psi$ decay modes

\begin{tabular}{|c|c|c|}
\hline \multicolumn{3}{|r|}{ J/Psi decay modes } \\
\hline \multicolumn{3}{|c|}{ 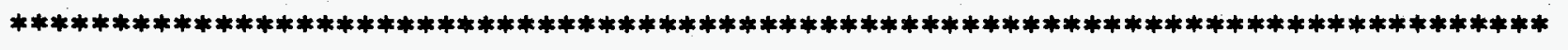 } \\
\hline JPSI & -1 & Defaults \\
\hline & $n=-1$ & $\begin{array}{r}\text { all decays allowed unless BOBO selected decay } 11,12,13 \\
\text { then only ete- and mu+mu- alloved }\end{array}$ \\
\hline & 0 & turn off all decays \\
\hline & 1 & all decays allowed \\
\hline & 2 & Psi $\rightarrow$ ete- only \\
\hline & 3 & $m u+m u-~ o n l y$ \\
\hline & 9 & $\theta+e-$ and mu+mu- only \\
\hline
\end{tabular}

JETD: Specify a file to read in JETSET decay information

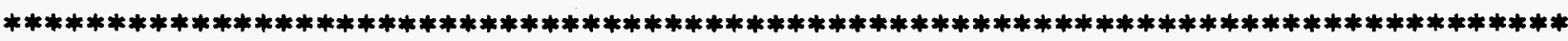

JETD 'filename' Specify a filo to read in JETSET decay information

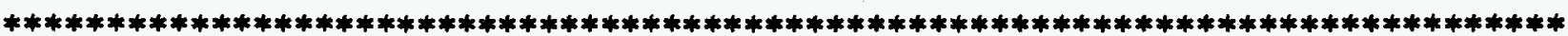
JETD ', Defaults

WARNIHG!!!!!

WARWING!!!!! You must use single quotes around the filename WARNING!!!!! e.g. JETD 'myfilo.dat' WARHING!!!!!

filename = Relative or absolute pathname that contains JETSET decay information a la LUUPDA. This info overrides the built-in decays. The file must contain the full decay table for a given particle but does not need to redefine every particle. ASLUND and BBKING users will recognize this as .newdat
} 


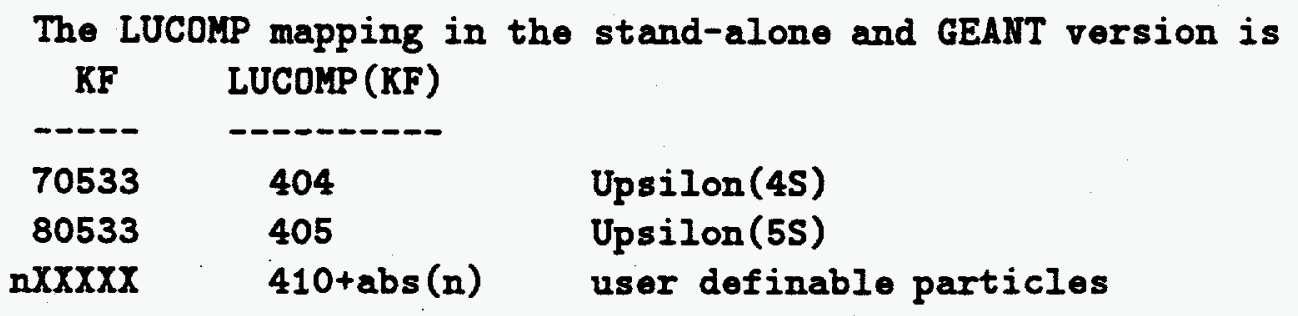

See Section 2.3.1 concerning LUCOMP in ASLUND

BEAM: Set the beam energies

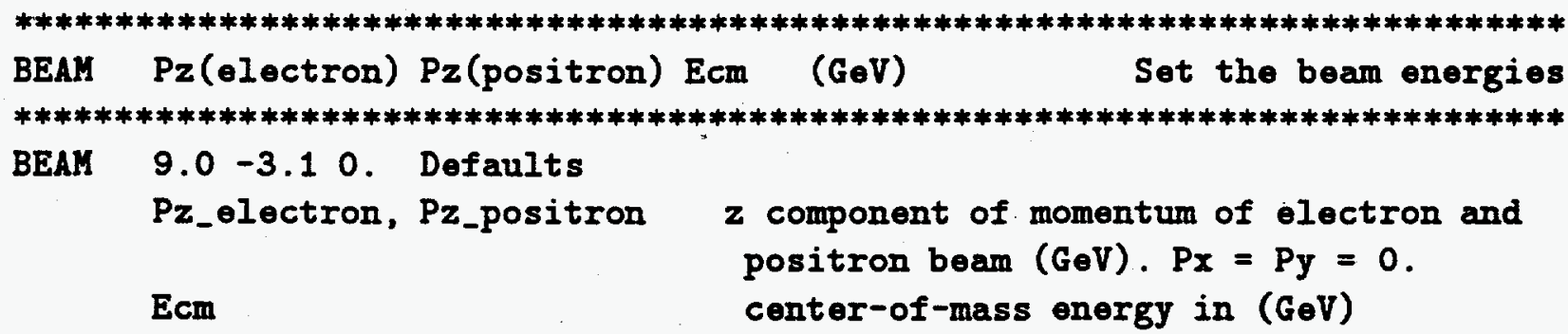

You may set EITHER the tro beam momenta OR one beam momentum and the center-of-mass energy. Set the unused quantity to zero and it vill automatically be filled in with the correct value. Note that the sign of the beam momenta is important.

SBEAM: Set the beam energy spread

******************************************************************************** SBEAM Sigma(electron) Sigma(positron) (GeV) Set the beam energy spread ****************************************************************************** SBEAM 0. 0. Defaults

The beam smearing is handled as in the native ASLUND generator, which smears the nominal beam energy by a gaussian for every event. For each event the center-of-mass is calculated and the mass of the Upsilon(4S) is set equal to this energy. The Upsilon(4S) is then generated with zero momentum. The boost of the final state is also based on the smeared energies on an event by event basis.

Warning, If the center-of-mass energy for a smeared event happens to be belor the mass threshold for BO production an empty event vill be 
generated. The actual threshold in JETSET is slightly higher than trice the BO mass.

VERT: Set the primary vertex location

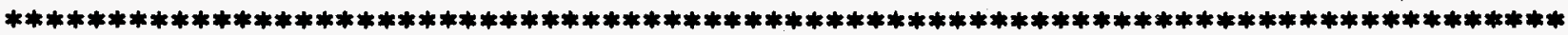
VERT $x y z$ (mm) Set the primary vertex location

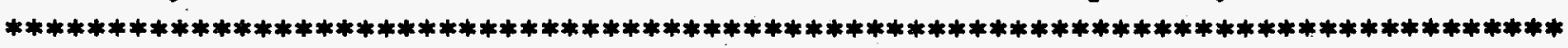
VERT 0. 0. 0. Defaults

The given coordinates, in millimeters, sets the primary vertex position

SVERT: Set the primary vertex spread

******************************************************************************** SVERT $d x d y d z$ (mm) Set the primary vertex spread

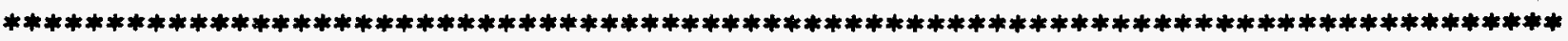
SVERT 0. 0. 0. Defaults

IVERT: Set the primary vertex smearing method

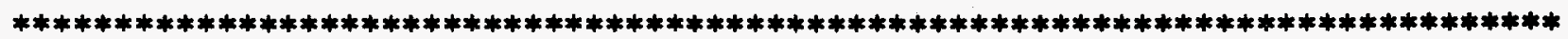
IVERT Ix Iy Iz

Set the primary vertex smearing method

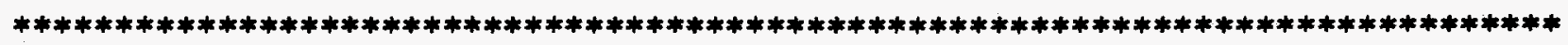
IVERT 000 Defaults $0=$ smear with gaussian (sigma given by SVERT) 1=smear with flat distribution (-delta,delta), (delta given by SVERT

GENP: Print event information on the terminal

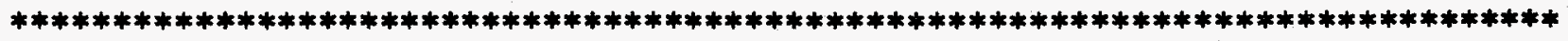
GENP IHEP ILUND IGPXINE IGPVERT Print event information on the terminal

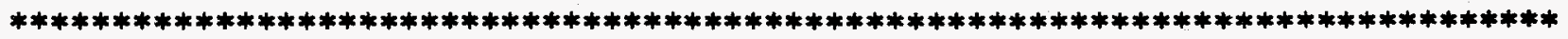
GENP

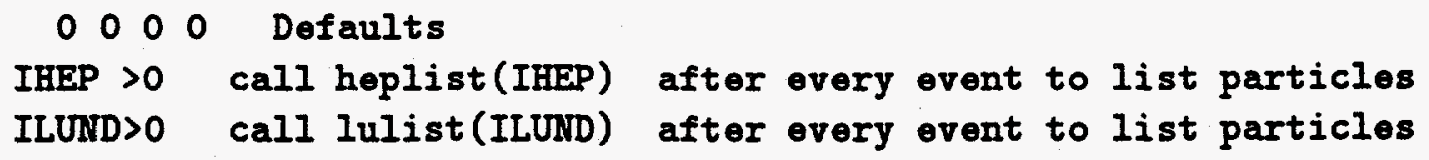


IGPKINE>0 call GPKINE(0) after every event to list GEANT particles IGPVERT>0 call GPVERT(0) after every event to list GEANT vertices IGPKINE and IGPVERT work only in GEANT programs

All four printouts can be activated simultaneously

JETO: Print the decay table for the listed particles

\begin{abstract}
****************************************************************************** JETO KF1 KF2 ... KF Print the decay table for the listed particles

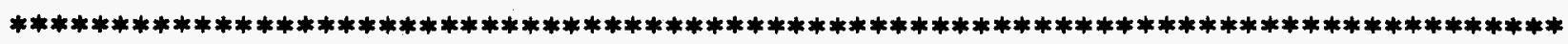
JETO 0 O $\quad \ldots$. 0 Defaults

For any non-zero JETSET particle code the decay table for that particle will be listed at the end of the initialization example: pass through the JETSET generator.

JETO 511443 \#ist the decay table for BO and J/PSI
\end{abstract}

RAN: Control the random number seeds

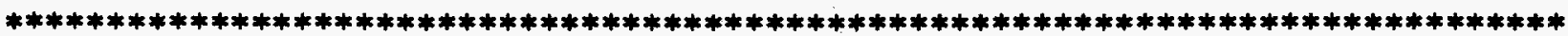
RAII Iseed1 Iseed2 Control the random number seeds

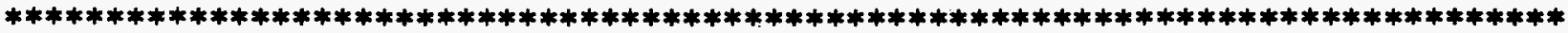
RAN 10 Defaults

This data card initializes the random number seed for the BEGET random number generator (RANECU). RAN behaves exactly like the RNDM data card of GEANT.

The preselected random number seeds (see belor) result in distinct sequences 1 billion numbers apart. Not all integer seeds result in a good or random sequence, so the authors recommend using the preselected ones.

Iseed2=0 Iseed1 can be a number betreen 1 and 100 that causes a preselected seed to initialize the random number generator.

Iseed2<>0 The random number generator will be initialized with seeds 
Iseed1 and Iseed2.

EVNT: Set the number of events to generate

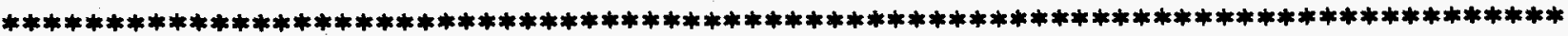

EVIT Nbeg_events

Set the number of events to generate

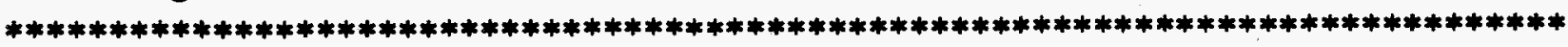

EVIT 1

Defaults

This data card merely sets a variable (Nbeg_events) in the BEGEI common block. It is up to the user to check it and call the BEGET subroutine the appropriate number of times.

ASLUND and BBSIM users should not use this card since event counting is already built in to those programs. 


\section{STDHEP Common Block}

The variables in the STDHEP common block are given in Table 5. The complete documentation including a table comparing the STDHEP particle codes with those of the Particle Data Group, JETSET, ISAJET, and HERWIG can be found in Reference [5].

Table 5: STDHEP common block variables.

\begin{tabular}{|c|c|}
\hline $\begin{array}{l}\text { NEVHEP } \\
\text { NHEP }\end{array}$ & $\begin{array}{l}\text { event number } \\
\text { number of entries in the event }\end{array}$ \\
\hline \multicolumn{2}{|r|}{ For each entry $i$} \\
\hline ISTHEP(i) & status code for particle \\
\hline IDHEP(i) & particle ID code \\
\hline JMOHEP $(1, \mathbf{i})$ & position of mother particle \\
\hline $\operatorname{JMOHEP}(2, \mathrm{i})$ & position of second mother particle \\
\hline $\operatorname{JDAHEP}(1, \mathrm{i})$ & position of first daughter \\
\hline JDAHEP $(2, \mathbf{i})$ & position of last daughter \\
\hline PHEP(1-3,i) & $p_{x}, p_{y}, p_{z}$ in $(\mathrm{GeV} / \mathrm{c})$ \\
\hline $\operatorname{PHEP}(4, \mathbf{i})$ & Energy (GeV) \\
\hline PHEP $(5, \mathrm{i})$ & $\operatorname{Mass}\left(\mathrm{GeV} / \mathrm{c}^{2}\right)$ \\
\hline VHEP(1-3,i) & $x, y, z$ or vertex $(\mathrm{mm})$ \\
\hline $\operatorname{VHEP}(4, \mathrm{i})$ & production time $(\mathrm{mm} / \mathrm{c})$ \\
\hline
\end{tabular}




\section{References}

[1] GEANT Detector Description and Simulation Tool,, CERN Program Library W5103, CERN, March 1994.

http : //asis01.cern.ch/cn/CNASDOC/geant/GEANTMAIN.html

[2] Originally written by Alan Weinstein, 1989.

T. Glanzman, W. Innes, S. Szena, and A. Snyder, ASLUND: The Desing of a Unix-based Group Software Project, BaB̄ar Note \# 116, November 1993.

T. Glanzman, W. Innes, S. Szena, and A. Snyder, Using ASLUND at SLAC, BaB̄ar Note \# 117, November 1993.

http://ww.slac.stanford. edu/BF/doc/PhysSim/aslund.txt

[3] T. Sjöstrand, JETSET 7.3, CERN-TH 6488/92, May 1992.

[4] S. Jadach and Z. Was, KORALB 2.1; An Upgrade with Tauola Library of Tau Decays, Comput. Phys. Comm. 64, 267(1991).

[5] Lynn Garren, STDHEP 1.05, FNAL PM0091, September, 1993. http:// wr.slac.stanford.edu/BF/doc/Miscellaneous/stdhep.ps

[6] FFREAD Format Free Input Processing, CERN Program Library I302, http ://asis01.cern.ch/cn/CNASDOC/ww/ffread/ffmain/ffmain.html

[7] Letter of Intent for the Study of CP Violation and Heavy Flavor Physics at PEP-II, BaBar Collaboration, SLAC-442, June 1994.

[8] HBOOK Reference Manual, CERN Program Library Y250, http ://asis01. cern. ch/cn/CHASDOC/hbook/HBOOKMAIN.html 